\title{
Design of two-channel linear-phase QMF banks based on real IIR all-pass filters
}

\author{
J.-H. Lee and Y.-H. Yang
}

\begin{abstract}
The design of two-channel linear-phase quadrature mirror filter (QMF) banks constructed by real infinite impulse response (IIR) digital all-pass filters is considered. The design problem is appropriately formulated to result in a simple optimisation problem. Using a variant of Karmarkar's algorithm, the optimisation problem can be efficiently solved through a frequency sampling and iterative approximation method to find the real coefficients for the IIR digital all-pass filters. The resulting two-channel QMF banks possess an approximately linear phase response without magnitude distortion. The effectiveness of the proposed technique is achieved by forming an appropriate Chebyshev approximation of the desired phase response and then finding its solution from a linear subspace in a few iterations. Finally, several simulation examples are presented for illustration and comparison.
\end{abstract}

\section{Introduction}

For many communication and signal processing systems, quadrature mirror filter (QMF) banks have been widely used to achieve the goals of subband coding and short-time spectral analysis [1-4]. In these applications, a QMF bank is employed to decompose a signal into subbands and the subband signals in the analysis system are decimated by an integer equal to the number of subbands. Moreover, twochannel QMF banks can easily be used for constructing $M$-channel QMF banks based on a tree structure. Hence, it is worth exploiting the design problem of two-channel QMF banks with linear phase characteristics.

Several techniques have been presented for designing two-channel QMF banks with IIR analysis filters and approximately linear phase based on the least-squares $\left(L_{2}\right)$ error criteria [5-12]. These IIR QMF banks are designed with the linear phase property imposed on the analysis filters. In contrast, a technique has been proposed in [13] for designing an IIR QMF bank with arbitrary group delay optimal in the minimax $\left(L_{\infty}\right)$ sense. Recently, the design results for IIR linear-phase QMF banks based on real allpass sections and complex all-pass sections have been reported in [9-12] and [14,15], respectively. The main advantage of using all-pass sections is that the designed IIR QMF banks can possess approximately linear phase response without magnitude distortion.

This paper presents a technique based on a variant of Karmarkar's algorithm [16] for the optimal design of twochannel linear-phase QMF banks using real IIR digital allpass filters (DAFs). The design problem is formulated by using the minimax error criteria on the phase approximation

\section{(C) IEE, 2003}

IEE Proceedings online no. 20030699

doi: 10.1049/ip-vis:20030699

Paper first received 23rd September 2002 and in revised form 11th April 2003

The authors are with the Department of Electrical Engineering, National Taiwan University, Taipei, 106, Taiwan, Republic of China to obtain an appropriate objective function that leads to a nonlinear optimisation problem. To effectively tackle the resulting nonlinear minimax optimisation problem, we utilise a nonlinear minimax algorithm [17] to result in a sequence of linear Chebyshev approximation problems. Each of the linear Chebyshev approximations provides the required increment for updating the filter coefficients during each iteration. As a result, the key operation of the proposed technique is to find the linear Chebyshev approximation of a desired phase response from a linear subspace related to the objective function. This can easily be solved by using a variant of Karmarkar's algorithm [16]. Several design examples showing the effectiveness of the proposed technique are also provided.

\section{Two-channel QMF banks with linear phase response}

Consider the two-channel filter bank with a system architecture shown in Fig. $1 . H_{0}(\mathrm{z})$ and $H_{1}(\mathrm{z})$ designate the low-pass and high-pass analysis filters, respectively, and $F_{0}(\mathrm{z})$ and $F_{1}(\mathrm{z})$ designate the low-pass and high-pass synthesis filters, respectively. We can easily show that the input-output relationship in the Z-transform is given by

$$
\begin{aligned}
\hat{X}(z)= & \frac{1}{2}\left[H_{0}(z) F_{0}(z)+H_{1}(z) F_{1}(z)\right] X(z) \\
& +\frac{1}{2}\left[H_{0}(-z) F_{0}(z)+H_{1}(-z) F_{1}(z)\right] X(-z)
\end{aligned}
$$

The first term of (1) represents a linear shift-invariant system response, which is the desired signal translation from $x(n)$ to $\hat{x}(n)$, and the second term represents the aliasing error due to the change of sampling rate in the filter bank. Setting the synthesis filters $F_{0}(z)=H_{1}(-z)$ and $F_{1}(z)=$ $-H_{0}(z)$ eliminates the aliasing term. As the mirror-image symmetry about the frequency $\omega=\pi / 2$ exists between $H_{0}(z)$ and $H_{1}(z)$, we have $H_{0}(z)=H_{1}(-z)$. Therefore (1) becomes

$$
\hat{X}(z)=\frac{1}{2}\left[H_{0}^{2}(z)-H_{0}^{2}(-z)\right] X(z)
$$




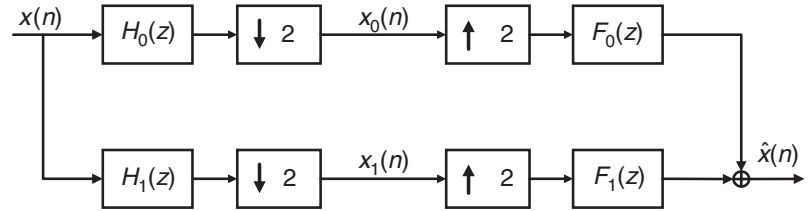

Fig. 1 Two-channel QMF bank

Setting $z=\exp (\mathrm{j} \omega)$ in (2), we get

$$
\hat{X}\left(\mathrm{e}^{\mathrm{j} \omega}\right)=\frac{1}{2}\left[H_{0}^{2}\left(\mathrm{e}^{\mathrm{j} \omega}\right)-H_{0}^{2}\left(\mathrm{e}^{\mathrm{j}(\omega+\pi)}\right)\right] X\left(\mathrm{e}^{\mathrm{j} \omega}\right)
$$

Let $T\left(\mathrm{e}^{\mathrm{j} \omega}\right)$ denote the frequency response of the QMF bank. Equation (3) reveals that producing a reconstructed signal $\hat{x}(n)$ that is a delayed replica of $x(n)$ requires

$$
T\left(\mathrm{e}^{\mathrm{j} \omega}\right)=H_{0}^{2}\left(\mathrm{e}^{\mathrm{j} \omega}\right)-H_{0}^{2}\left(\mathrm{e}^{\mathrm{j}(\omega+\pi)}\right)=\mathrm{e}^{-\mathrm{j} g_{d} \omega} \text { for all } \omega
$$

where $g_{d}$ is the system delay of the QMF bank. This imposes constraints not only that $H_{0}(z)$ should be an ideal low-pass filter, but also that its behaviour for all $\omega$ should satisfy the condition given in (4). The designs of QMF banks using conventional FIR or IIR structures for $H_{0}(z)$ usually induce both magnitude and phase distortions.

\section{Problem formulation of two-channel QMF bank design}

Here, we consider the two-channel QMF bank with analysis and synthesis structures shown by Figs. 2 and 3 , respectively, where $A_{1}\left(z^{2}\right)$ and $A_{2}\left(z^{2}\right)$ are two real IIR DAFs. The main advantage of using this kind of QMF bank is that the design problem can be focused on the phase approximation without magnitude distortion $[11,12]$. In the following, we briefly describe how to construct this twochannel QMF bank from the conventional structure shown in Fig. 1. By setting the analysis filters $H_{0}(z)$ and $H_{1}(z)$ of Fig. 1 to

$$
H_{0}(z)=\frac{B_{1}(z)+B_{2}(z)}{2} \text { and } H_{1}(z)=\frac{B_{1}(z)-B_{2}(z)}{2}
$$

respectively, where $B_{i}(z)$ are two real IIR DAPs with orders equal to $N_{i}$ and frequencies given by

$$
B_{i}\left(\mathrm{e}^{\mathrm{j} \omega}\right)=\mathrm{e}^{-\mathrm{j} N_{i} \omega} \frac{\sum_{n=0}^{N_{i}} a_{i}(n) \mathrm{e}^{\mathrm{j} n \omega}}{\sum_{n=0}^{N_{i}} a_{i}(n) \mathrm{e}^{-\mathrm{j} n \omega}}, \quad \text { for } i=1,2
$$

From (5), we have

$$
B_{1}(z)=H_{0}(z)+H_{1}(z) \text { and } B_{2}(z)=H_{0}(z)-H_{1}(z)
$$

Hence, $H_{0}(z)$ and $H_{1}(z)$ satisfy the all-pass complementary and power complementary properties. They are termed the doubly-complementary (DC) filter pair [18].

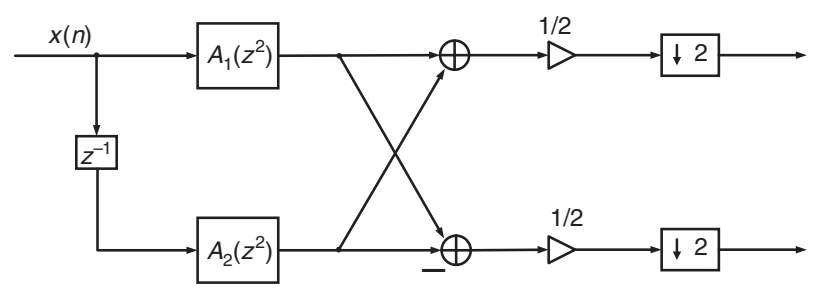

Fig. 2 Analysis system for DC-based QMF bank

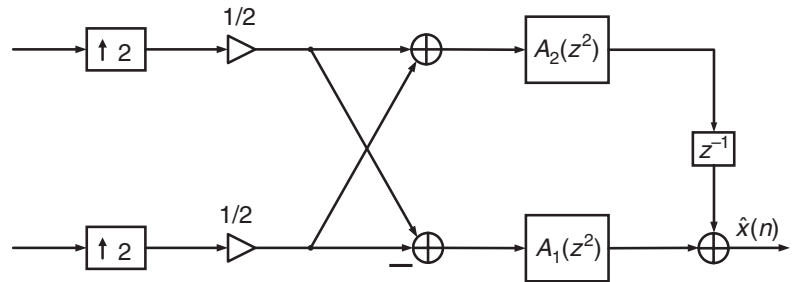

Fig. 3 Synthesis system for DC-based QMF bank

Moreover, the QMF property requires the condition $H_{0}(z)=H_{1}(-z)$. The resulting filter banks are termed DC-based QMF filter banks [9-12, 14, 15]. Hence, we have from (7) that

$$
B_{1}(z)=H_{0}(z)+H_{0}(-z)=2 h_{0}+2 h_{2} z^{-2}+\ldots=A_{1}\left(z^{2}\right)
$$

and

$$
\begin{aligned}
B_{2}(z) & =H_{0}(z)-H_{0}(-z)=z^{-1}\left[2 h_{1}+2 h_{3} z^{-2}+\ldots\right] \\
& =z^{-1} A_{2}\left(z^{2}\right)
\end{aligned}
$$

where $h_{i}, i=0,1, \ldots$, represent the impulse response coefficients of $H_{0}(z)$. Consequently, (5) can be rewritten as follows:

$$
\begin{aligned}
& H_{0}(z)=\frac{A_{1}\left(z^{2}\right)+z^{-1} A_{2}\left(z^{2}\right)}{2} \text { and } \\
& H_{1}(z)=\frac{A_{1}\left(z^{2}\right)-z^{-1} A_{2}\left(z^{2}\right)}{2}
\end{aligned}
$$

Substituting (9) into (4) yields the frequency response of the DC-based QMF bank as follows:

$$
T\left(\mathrm{e}^{\mathrm{j} \omega}\right)=\frac{1}{2} \mathrm{e}^{-\mathrm{j} \omega} A_{1}\left(\mathrm{e}^{\mathrm{j} 2 \omega}\right) A_{2}\left(\mathrm{e}^{\mathrm{j} 2 \omega}\right)
$$

Equation (10) reveals that the resulting QMF bank possesses perfect magnitude response, i.e. there is no magnitude distortion. Therefore, the design problem is to find real coefficients for the IIR DAFs $A_{1}\left(z^{2}\right)$ and $A_{2}\left(z^{2}\right)$ such that the resulting phase response $\operatorname{Arg}\left\{T\left(\mathrm{e}^{\mathrm{j} \omega}\right)\right\}$ of the QMF bank can approximate the desired phase characteristic in the minimax sense. From (6), we have the real IIR DAFs $A_{1}\left(z^{2}\right)$ and $A_{2}\left(z^{2}\right)$ with frequency responses given by

$$
A_{1}\left(\mathrm{e}^{\mathrm{j} 2 \omega}\right)=\mathrm{e}^{-\mathrm{j} 2 N_{1} \omega} \frac{\sum_{n=0}^{N_{1}} a_{1}(n) \mathrm{e}^{\mathrm{j} 2 n \omega}}{\sum_{n=0}^{N_{1}} a_{1}(n) \mathrm{e}^{-\mathrm{j} 2 n \omega}}=\mathrm{e}^{\mathrm{j} \theta_{1}(\omega)} \text { and }
$$

$$
A_{2}\left(\mathrm{e}^{\mathrm{j} 2 \omega}\right)=\mathrm{e}^{-\mathrm{j} 2 N_{2} \omega} \frac{\sum_{n=0}^{N_{2}} a_{2}(n) \mathrm{e}^{\mathrm{j} 2 n \omega}}{\sum_{n=0}^{N_{2}} a_{2}(n) \mathrm{e}^{-\mathrm{j} 2 n \omega}}=\mathrm{e}^{\mathrm{j} \theta_{2}(\omega)}
$$

respectively. Moreover, without loss of generality, both coefficients $a_{1}(0)$ and $a_{2}(0)$ can be set to one. Then, the phase responses $\theta_{i}(\omega), i=1,2$, are given by

$$
\begin{aligned}
\theta_{i}(\omega) & =-2 N_{i} \omega-2 \phi_{i}(\omega) \\
& =-2 N_{i} \omega-2 \tan ^{-1}\left\{\frac{-\sum_{n=1}^{N_{i}} a_{i}(n) \sin (2 n \omega)}{1+\sum_{n=1}^{N_{i}} a_{i}(n) \cos (2 n \omega)}\right\}
\end{aligned}
$$


Substituting (11) into (9) yields

$$
\begin{aligned}
H_{0}\left(\mathrm{e}^{\mathrm{j} \omega}\right)= & \frac{1}{2}\left[\mathrm{e}^{\mathrm{j} \theta_{1}(\omega)}+\mathrm{e}^{-\mathrm{j} \omega} \mathrm{e}^{\mathrm{j} \theta_{2}(\omega)}\right] \\
= & \exp \left(\mathrm{j} \frac{\theta_{1}(\omega)+\theta_{2}(\omega)-\omega}{2}\right) \\
& \times \cos \left(\frac{\theta_{1}(\omega)-\theta_{2}(\omega)+\omega}{2}\right)
\end{aligned}
$$

and

$$
\begin{aligned}
H_{1}\left(\mathrm{e}^{\mathrm{j} \omega}\right)= & \frac{1}{2}\left[\mathrm{e}^{\mathrm{j} \theta_{1}(\omega)}-\mathrm{e}^{-\mathrm{j} \omega} \mathrm{e}^{\mathrm{j} \theta_{2}(\omega)}\right] \\
= & \mathrm{j} \exp \left(\mathrm{j} \frac{\theta_{1}(\omega)+\theta_{2}(\omega)-\omega}{2}\right) \\
& \times \sin \left(\frac{\theta_{1}(\omega)-\theta_{2}(\omega)+\omega}{2}\right)
\end{aligned}
$$

In order to guarantee that $H_{0}(z)$ and $H_{1}(z)$ are linearphase low-pass and high-pass filters, respectively, we can impose the following conditions on $\theta_{i}(\omega), i=1,2$, based on (13) and (14):

Case (i): For $N_{1}=N_{2}=N$ :

$$
\begin{aligned}
& \left\{\begin{array}{l}
\theta_{1}(\omega)=-2 N \omega-\omega / 2 \\
\theta_{2}(\omega)=-2 N \omega+\omega / 2
\end{array}\right\}, \quad \text { for } 0 \leqq \omega \leqq \omega_{p} \\
& \left\{\begin{array}{l}
\theta_{1}(\omega)=-2 N \omega-\omega / 2+\pi / 2 \\
\theta_{2}(\omega)=-2 N \omega+\omega / 2-\pi / 2
\end{array}\right\}, \quad \text { for } \omega_{s} \leqq \omega \leqq \pi
\end{aligned}
$$

Case (ii): For $N_{1}=N_{2}+1$ :

$$
\begin{aligned}
& \left\{\begin{array}{l}
\theta_{1}(\omega)=-2 N_{1} \omega+\omega / 2 \\
\theta_{2}(\omega)=-2 N_{2} \omega-\omega / 2
\end{array}\right\}, \quad \text { for } 0 \leqq \omega \leqq \omega_{p} \\
& \left\{\begin{array}{l}
\theta_{1}(\omega)=-2 N_{1} \omega+\omega / 2-\pi / 2 \\
\theta_{2}(\omega)=-2 N_{2} \omega-\omega / 2+\pi / 2
\end{array}\right\}, \quad \text { for } \omega_{s} \leqq \omega \leqq \pi
\end{aligned}
$$

where $\omega_{p}$ and $\omega_{s}$ are the passband and stopband edge frequencies of $H_{0}(z)$, respectively. Equations (15) and (16) reveal that the above conditions also satisfy the following stability constraints for the real IIR DAFs $A_{i}\left(z^{2}\right)[15,18]$ : $\theta_{i}(\omega)$ is monotonically decreasing and $\theta_{i}(\pi)=\theta_{i}(0)-$ $2 N_{i} \pi$, for $i=1,2$.

Next, from (10), (15) and (16), we note that the frequency response of the DC-based QMF bank becomes

$$
\begin{aligned}
T\left(\mathrm{e}^{\mathrm{j} \omega}\right) & =\frac{1}{2} \exp \left[\mathrm{j}\left(-\omega+\theta_{1}(\omega)+\theta_{2}(\omega)\right)\right] \\
& =\frac{1}{2} \exp \left[-\mathrm{j}\left(2 N_{1}+2 N_{2}+1\right) \omega\right]
\end{aligned}
$$

Equation (17) reveals that the resulting DC-based QMF bank possesses a linear phase response with group delay $g_{d}=2 N_{1}+2 N_{2}+1$ and without magnitude distortion. As a result, the design problem can be formulated as follows. Find filter coefficients $a_{i}(n)$ of the real IIR DAPs $A_{i}\left(z^{2}\right)$, $i=1,2$, and $n=0,1,2, \ldots, N_{i}$, such that the resulting phase responses $\theta_{i}(\omega)$ given by (12) approximate the desired phase responses as shown by (15) and (16) in the minimax sense. Comparing (12), (15) and (16), one can obtain the corresponding design problems for case (i) and case (ii), respectively, as follows:
Case (i):

$$
\text { Minimise }\left\|-\tan ^{-1}\left\{\frac{\sum_{n=0}^{N_{i}} a_{i}(n) \sin (2 n \omega)}{1+\sum_{n=0}^{N_{i}} a_{i}(n) \cos (2 n \omega)}\right\}-\phi_{i}(\omega)\right\|
$$

where $\phi_{i}(\omega)$ are given by

$$
\begin{gathered}
\left\{\begin{array}{c}
\phi_{1}(\omega)=\frac{\omega}{4}, 0 \leq \omega \leq \omega_{p} \\
\phi_{1}(\omega)=\frac{\omega}{4}-\frac{\pi}{4}, \omega_{s} \leq \omega \leq \pi
\end{array}\right\} \text { and } \\
\left\{\begin{array}{c}
\phi_{2}(\omega)=-\frac{\omega}{4}, 0 \leq \omega \leq \omega_{p} \\
\phi_{2}(\omega)=-\frac{\omega}{4}+\frac{\pi}{4}, \omega_{s} \leq \omega \leq \pi
\end{array}\right\}
\end{gathered}
$$

Case (ii):

$$
\text { Minimise }\left\|-\tan ^{-1}\left\{\frac{\sum_{n=0}^{N_{i}} a_{i}(n) \sin (2 n \omega)}{1+\sum_{n=0}^{N_{i}} a_{i}(n) \cos (2 n \omega)}\right\}-\phi_{i}(\omega)\right\|
$$

where $\phi_{i}(\omega)$ are given by

$$
\begin{aligned}
& \left\{\begin{array}{c}
\phi_{1}(\omega)=-\frac{\omega}{4}, 0 \leq \omega \leq \omega_{p} \\
\phi_{1}(\omega)=-\frac{\omega}{4}+\frac{\pi}{4}, \omega_{s} \leq \omega \leq \pi
\end{array}\right\} \text { and } \\
& \left\{\begin{array}{c}
\phi_{2}(\omega)=\frac{\omega}{4}, 0 \leq \omega \leq \omega_{p} \\
\phi_{2}(\omega)=\frac{\omega}{4}-\frac{\pi}{4}, \omega_{s} \leq \omega \leq \pi
\end{array}\right\}
\end{aligned}
$$

and $\|\mathrm{x}\|$ denotes the Chebyshev norm. Let

$$
M_{i}(\omega)=\frac{\sum_{n=0}^{N_{i}} a_{i}(n) \sin (2 n \omega)}{1+\sum_{n=0}^{N_{i}} a_{i}(n) \cos (2 n \omega)}
$$

Then, (18) and (20) can be rewritten as

$$
\text { Minimise }\left\|\tan ^{-1}\left(M_{i}(\omega)\right)+\tan ^{-1} \tan \left(\phi_{i}(\omega)\right)\right\|
$$

Using the addition formula of inverse tangents, we obtain

$$
\text { Minimise }\left\|\tan ^{-1}\left\{\frac{M_{i}(\omega)+\tan \left(\phi_{i}(\omega)\right)}{1-M_{i}(\omega) \tan \left(\phi_{i}(\omega)\right)}\right\}\right\|
$$

From (24), an equivalent minimisation problem can be obtained by dropping the inverse tangent operation as follows:

$$
\text { Minimise }\left\|\frac{M_{i}(\omega)+\tan \left(\phi_{i}(\omega)\right)}{1-M_{i}(\omega) \tan \left(\phi_{i}(\omega)\right)}\right\|
$$

because the inverse tangent function is a monotonic function. Performing some algebraic manipulation on (25) yields the following minimisation problem

Minimise $\left\|\frac{\sum_{n=1}^{N_{i}} a_{i}(n)\left[\sin (2 n \omega)+\varphi_{i}(\omega) \cos (2 n \omega)\right]+\varphi_{i}(\omega)}{\sum_{n=1}^{N_{i}} a_{i}(n)\left[\cos (2 n \omega)-\varphi_{i}(\omega) \sin (2 n \omega)\right]+1}\right\|$

where $\varphi_{i}(\omega)=\tan \left(\phi_{i}(\omega)\right)$, for $i=1,2$. Therefore, the overall design problem is to find the optimal coefficients $a_{i}(n), n=1,2, \ldots, N_{i}, i=1,2$, for the minimisation problem of (26). However, (26) represents a highly nonlinear minimisation problem. Directly solving (26) is not an easy task. 


\section{Proposed design technique}

In this Section, we present a design technique based on the nonlinear minimax algorithm of [17] in conjunction with a variant of Karmarkar's algorithm [16] for solving the resulting minimisation problem of (26). This is through a frequency sampling and iterative approximation scheme to find the optimal coefficients $a_{i}(n), n=1,2, \ldots, N_{i}, i=1,2$, for the real IIR DAFs shown by (11). At the $k$ th iteration, we construct the following function:

$$
\operatorname{Apx}\left(\boldsymbol{a}_{k}, \omega\right)=\frac{\sum_{n=1}^{N_{i}} a_{i k}(n)\left[\sin (2 n \omega)+\varphi_{i}(\omega) \cos (2 n \omega)+\varphi_{i}(\omega)\right.}{\sum_{n=1}^{N_{i}} a_{i k}(n)\left[\cos (2 n \omega)-\varphi_{i}(\omega) \sin (2 n \omega)\right]+1}
$$

and the $N_{i} \times 1$ gradient vector of $\operatorname{Apx}\left(\boldsymbol{a}_{k}, \omega\right)$ given by

$$
\nabla \operatorname{Apx}\left(\boldsymbol{a}_{k}, \omega\right)=\left[\psi_{1}(\omega) \psi_{2}(\omega) \ldots \psi_{N i}(\omega)\right]^{\mathrm{T}}
$$

where $\psi_{n}(\omega)$ represents the $n$th gradient component of $\operatorname{Apx}\left(\boldsymbol{a}_{k}, \omega\right)$ and is given as follows:

$$
\begin{aligned}
\psi_{n}(\omega) & =\frac{\partial \operatorname{Apx}\left(\boldsymbol{a}_{k}, \omega\right)}{\partial a_{i}(n)} \\
= & \left(\left[\sin (2 n \omega)+\varphi_{i}(\omega) \cos (2 n \omega)\right]\right. \\
& \left.\times\left(\sum_{n^{\prime}=1}^{N_{i}} a_{i k}\left(n^{\prime}\right)\left[\cos \left(2 n^{\prime} \omega\right)-\varphi_{i}(\omega) \sin \left(2 n^{\prime} \omega\right)\right]+1\right)\right) / \\
& \left(\sum_{n^{\prime}=1}^{N_{i}} a_{i k}\left(n^{\prime}\right)\left[\cos \left(2 n^{\prime} \omega\right)-\varphi_{i}(\omega) \sin \left(2 n^{\prime} \omega\right)\right]+1\right)^{2} \\
& -\left(\left[\cos (2 n \omega)-\varphi_{i}(\omega) \sin (2 n \omega)\right]\right. \\
& \left.\times\left(\sum_{n^{\prime}=1}^{N_{i}} a_{i k}\left(n^{\prime}\right)\left[\sin \left(2 n^{\prime} \omega\right)+\varphi_{i}(\omega) \cos \left(2 n^{\prime} \omega\right)\right]+\varphi_{i}(\omega)\right)\right) \\
& \left(\sum_{n^{\prime}=1}^{N_{i}} a_{i k}\left(n^{\prime}\right)\left[\cos \left(2 n^{\prime} \omega\right)-\varphi_{i}(\omega) \sin \left(2 n^{\prime} \omega\right)\right]+1\right)^{2}
\end{aligned}
$$

for $n=1,2, \ldots, N_{i}$. Let $\Omega=\left\{\omega_{1}=0, \omega_{2} \ldots, \omega_{L}=\pi\right\}$ be a dense grid of frequencies uniformly distributed in the range from $\omega=0$ to $\omega=\pi$. The design process of the proposed technique is then performed on $\Omega$. Next, a linearisation scheme is utilised to approximate the related phase error of (27) due to a perturbation in the filter coefficient vector in the linear subspace spanned by the gradient matrix associated with $\operatorname{Apx}\left(\boldsymbol{a}_{k}, \omega\right)$. As a result, the approximation for minimising the above peak phase error as shown in (26) can be formulated as finding the increment $\delta \boldsymbol{a}$ of the filter coefficient vector $\boldsymbol{a}=\left[a_{i}(1), a_{i}(2), \ldots, a_{i}\left(N_{i}\right)\right]^{\mathrm{T}}, i=1,2$, at the $k$ th iteration such that $\|-\operatorname{Apx}\left(\boldsymbol{a}_{k}, \omega_{l}\right)-\delta \boldsymbol{a}^{\mathrm{T}} \nabla \operatorname{Apx}\left(\boldsymbol{a}_{k}\right.$, $\left.\omega_{l}\right) \|$ is minimised. Let $\delta \boldsymbol{a}$ be expressed as $\delta \boldsymbol{a}=\left[\delta a_{i}(1) \delta a_{i}\right.$ (2) $\left.\ldots \delta a_{i}\left(N_{i}\right)\right]^{\mathrm{T}}$, the expression $\|-\operatorname{Apx}\left(\boldsymbol{a}_{k}, \omega_{l}\right)-\delta \boldsymbol{a}^{\mathrm{T}} \nabla \mathrm{Apx}$ $\left(\boldsymbol{a}_{k}, \omega_{l}\right) \|$ can be rewritten as follows:

$$
\left\|-\operatorname{Apx}\left(\boldsymbol{a}_{k}, \omega_{l}\right)-\sum_{n=l}^{N_{i}} \delta a_{i k}(n) \psi_{n}\left(\omega_{l}\right)\right\|
$$

As a result, finding the solution to minimise (30) is equivalent to calculating $\delta \boldsymbol{a}$ in an $N_{i}$-dimensional linear subspace $\Psi$ spanned by the set of basis functions $\left\{\psi_{1}(\omega), \psi_{2}(\omega), \ldots, \psi_{N_{i}}(\omega)\right\}$ to minimise (30). We note that the minimisation of (30) can be achieved by performing the following minimisation problem

$$
\text { Minimise }\left\|\boldsymbol{U} \delta \boldsymbol{a}_{k}-\boldsymbol{s}\right\|
$$

where $\boldsymbol{U}$ is a $L \times N_{i}$ matrix with entries given by $\psi_{n}\left(\omega_{l}\right)$, $1 \leq l \leq L, 1 \leq n \leq N_{i}$ and $s$ is a $L \times 1$ vector with the $l$ th entry given by $s(l)=-\operatorname{Apx}\left(\boldsymbol{a}_{k}, \omega_{l}\right), 1 \leqq l \leqq L$, and $\delta \boldsymbol{a}_{k}$ represents the increment computed for updating the coefficient vector $\boldsymbol{a}_{k}$. Equation (31) represents an equivalent form of the following linear Chebyshev minimisation problem:

$$
\begin{array}{ll}
\text { Minimise } & \varepsilon \\
\text { Subject to } & \left|\boldsymbol{U} \delta \boldsymbol{a}_{k}-\boldsymbol{s}\right| \leqq \varepsilon \mathbf{1},
\end{array}
$$

where $\mathbf{1}$ is a $L \times 1$ vector with all entries equal to 1 . The minimisation problem of (32) can be further rewritten as

$$
\begin{array}{ll}
\text { Minimise } & \varepsilon \\
\text { Subject to } & \left\{\boldsymbol{U} \delta \boldsymbol{a}_{k}-\varepsilon 1 \leq \boldsymbol{s},-\boldsymbol{U} \delta \boldsymbol{a}_{k}-\varepsilon 1 \leqq-\boldsymbol{s}\right.
\end{array}
$$

This leads to the standard dual form of a linear programming (LP) problem as follows:

$$
\begin{array}{ll}
\text { Maximise } & \boldsymbol{b}^{\mathrm{T}} \boldsymbol{w} \\
\text { Subject to } & \boldsymbol{A}^{\mathrm{T}} \boldsymbol{w} \leqq \boldsymbol{c}
\end{array}
$$

where

$$
\begin{aligned}
& \boldsymbol{w}=\left[\begin{array}{ll}
\delta \boldsymbol{a}_{k} & \varepsilon
\end{array}\right]^{\mathrm{T}}, \boldsymbol{b}=\left[\begin{array}{ll}
\mathbf{0}^{\mathrm{T}} & -1
\end{array}\right]^{\mathrm{T}}, \\
& \boldsymbol{c}=\left[\begin{array}{ll}
\boldsymbol{s}^{\mathrm{T}} & -\boldsymbol{s}^{\mathrm{T}}
\end{array}\right]^{\mathrm{T}}, \boldsymbol{A}=\left[\begin{array}{cc}
\boldsymbol{U}^{\mathrm{T}} & -\boldsymbol{U}^{\mathrm{T}} \\
-\mathbf{1}^{\mathrm{T}} & -\mathbf{1}^{\mathrm{T}}
\end{array}\right]
\end{aligned}
$$

where $\mathbf{0}$ is a $N_{i} \times 1$ vector with all entries equal to 0 . Using definition 3.1 of [19], we can easily obtain the standard primal form of (34) as follows:

$$
\begin{array}{ll}
\text { Minimise } & \boldsymbol{c}^{\mathrm{T}} \boldsymbol{x} \\
\text { Subject to } & \boldsymbol{A} \boldsymbol{x}=\boldsymbol{b}, \boldsymbol{x} \geqq 0
\end{array}
$$

where $\boldsymbol{w}$ and $\boldsymbol{x}$ respectively denote the dual variable and the corresponding primal variable for the above LP problems. In the following, we describe how to adopt the primal-form affine scaling variant of Karmarkar's algorithm (the PAS algorithm) of [16] for solving (36).

Assume that an initial solution $\boldsymbol{x}$ which satisfies the constraints is given. Then, $\boldsymbol{x}$ is mapped into a vector $\boldsymbol{y}^{0}$ with all entries equal to one as follows:

$$
\boldsymbol{y}=\boldsymbol{D}_{x}^{-1} \boldsymbol{x}
$$

where $\boldsymbol{D}_{x}$ denotes a diagonal matrix containing the entries of $\boldsymbol{x}$. Based on the mapping, we create

$$
\underline{\boldsymbol{A}}=\boldsymbol{A} \boldsymbol{D}_{x} \text { and } \underline{\boldsymbol{c}}=\boldsymbol{D}_{x} \boldsymbol{c}
$$

To satisfy the equality constraints, we project $\boldsymbol{c}$ onto the null space of $\underline{\boldsymbol{A}}$ to obtain

$$
\underline{c}_{\mathrm{p}}=\boldsymbol{P} \underline{\boldsymbol{c}},
$$

where

$$
\boldsymbol{P}=\boldsymbol{I}-\underline{\boldsymbol{A}}^{\mathrm{T}}\left(\underline{\boldsymbol{A}}^{\mathrm{T}}\right)^{-1} \underline{\boldsymbol{A}}
$$

denotes the projection operator. Next, we move from the initial $\boldsymbol{y}$ to $\boldsymbol{y}_{1}$ in the direction $-\underline{c}_{\mathrm{p}}$ to reduce the transformed objective function in the maximum rate according to

$$
\boldsymbol{y}^{1}=\mathbf{1}-\xi \frac{\underline{\boldsymbol{c}}_{\mathrm{p}}}{\max _{\mathrm{i}}\left(\boldsymbol{e}_{\mathrm{i}}^{\mathrm{T}} \underline{\boldsymbol{c}}_{\mathrm{p}}\right)}
$$


where the required step size $\xi \in(0,1)$ is chosen so that $\boldsymbol{y}^{1}>\mathbf{0}, \mathbf{1}$ represents a vector with appropriate size and all entries equal to one, and $\boldsymbol{e}_{\mathrm{i}}$ is a vector with appropriate size and the $i$ th entry equal to one and the others equal to zero. After obtaining $\boldsymbol{y}^{1}$, we then find a new feasible solution $\boldsymbol{x}^{1}$ for (36) by performing the inverse mapping.

Consider the projection operator given by (40). Substituting (38) into (40) yields

$$
\boldsymbol{P}=\boldsymbol{I}-\boldsymbol{D}_{x} \boldsymbol{A}^{\mathrm{T}}\left(\boldsymbol{A} \boldsymbol{D}_{x}^{2} \boldsymbol{A}^{\mathrm{T}}\right)^{-1} \boldsymbol{A} \boldsymbol{D}_{x}
$$

Accordingly, we have from (38), (39), and (42) that

$$
\underline{c}_{\mathrm{p}}=\boldsymbol{D}_{x}\left[\boldsymbol{I}-\boldsymbol{A}^{\mathrm{T}}\left(\boldsymbol{A} \boldsymbol{D}_{x}^{2} \boldsymbol{A}^{\mathrm{T}}\right)^{-1} \boldsymbol{A} \boldsymbol{D}_{x}^{2}\right] \boldsymbol{c}
$$

Next, we define the following two vectors

$$
\boldsymbol{w}=\left(\boldsymbol{A} \boldsymbol{D}_{x}^{2} \boldsymbol{A}^{\mathrm{T}}\right)^{-1} \boldsymbol{A} \boldsymbol{D}_{x}^{2} \boldsymbol{c} \text { and } \boldsymbol{r}=\boldsymbol{c}-\boldsymbol{A}^{\mathrm{T}} \boldsymbol{w}
$$

where $\boldsymbol{w}$ represents the dual variable vector associated with the primal variable vector $\boldsymbol{x}$. Hence, the primal variable $\boldsymbol{x}^{k+1}$ at the $(k+1)$ th iteration is found by the following equation

$$
\boldsymbol{x}^{k+1}=\boldsymbol{x}^{k}-\frac{\xi}{\gamma} \boldsymbol{d}_{x}
$$

where

$$
\begin{aligned}
\boldsymbol{d}_{x}=\boldsymbol{D}_{x}^{2} \boldsymbol{r} \text { and } \gamma & =\max _{i}\left(\boldsymbol{e}_{i}^{\mathrm{T}} \underline{\boldsymbol{c}}_{\mathrm{p}}\right)=\max _{i}\left(\boldsymbol{e}_{i}^{\mathrm{T}} \boldsymbol{D}_{x} \boldsymbol{r}\right) \\
& =\max _{i}\left(\boldsymbol{e}_{i}^{\mathrm{T}} \boldsymbol{D}_{x}^{2} \boldsymbol{r} / x_{i}\right)
\end{aligned}
$$

with $x_{i}$ being the $i$ th entry of $\boldsymbol{x}$.

\subsection{Determination of initial guess for the filter coefficient vector}

To initiate the design process, we have to make an initial guess $\boldsymbol{a}_{0}$ for the filter coefficient vector $\boldsymbol{a}$ according to the nonlinear minimax algorithm of [17]. An appropriate manner is to minimize the squared value of the numerator of $\operatorname{Apx}(\boldsymbol{a}, \omega)$ given by (27), i.e. minimise

$$
\sum_{l=1}^{L}\left(\sum_{n=1}^{N_{i}} a_{i}(n)\left[\sin \left(2 n \omega_{l}\right)+\varphi_{i}\left(\omega_{l}\right) \cos \left(2 n \omega_{l}\right)\right]+\varphi_{i}\left(\omega_{l}\right)\right)^{2}
$$

To solve this minimisation problem, we use a simple approach as follows. Let $\boldsymbol{X}$ be a $L \times N_{i}$ matrix whose entries are given by

$$
\begin{gathered}
X(l, n)=\sin \left(2 n \omega_{l}\right)+\varphi_{i}\left(\omega_{l}\right) \cos \left(2 n \omega_{l}\right), \\
1 \leq l \leq L, 1 \leq n \leq N_{i}
\end{gathered}
$$

Then, it is easy to show that minimising (47) yields the required initial guess $\boldsymbol{a}_{0}$ as follows:

$$
\boldsymbol{a}_{0}=\left(\boldsymbol{X}^{\mathrm{T}} \boldsymbol{X}\right)^{-1} \boldsymbol{X}^{\mathrm{T}} \boldsymbol{q}
$$

where the $L \times 1$ vector $\boldsymbol{q}$ has entries given by

$$
q(l)=-\varphi_{i}\left(\omega_{l}\right), \quad 1 \leq l \leq L
$$

After finding the appropriate initial guess $\boldsymbol{a}_{0}$ and setting the initial filter coefficient increment to a zero vector, we present an iterative procedure based on the above presentation to compute the filter coefficient vector $\boldsymbol{a}$ during the design process.

\subsection{Iterative procedure}

Step 1: Determine the design parameters: the orders $N_{1}$ and $N_{2}$, passband edge frequency $\omega_{p}$ and stopband edge frequency $\omega_{s}$. Make an initial guess $\boldsymbol{a}_{0}$ for the filter coefficient vector $\boldsymbol{a}=\left[a_{i}(1), a_{i}(2), \ldots, a_{i}\left(N_{i}\right)\right]^{\mathrm{T}}, i=1,2$, from (49) and set the iteration number $k=0$.

Step 2: Perform a test to stop the iteration process. We set a stopping criterion as follows: if $\mid\left(\max \left\{\left|\boldsymbol{U} \delta \boldsymbol{a}_{k}-\boldsymbol{s}\right|\right\}-\right.$ $\left.\max \left\{\left|\boldsymbol{U} \delta \boldsymbol{a}_{k+1}-\boldsymbol{s}\right|\right\}\right) / \max \left\{\left|\boldsymbol{U} \delta \boldsymbol{a}_{k}-\boldsymbol{s}\right|\right\} \mid \leqq v$, then the design process is terminated and the filter coefficient vector $\boldsymbol{a}_{k}$ obtained contains the designed filter coefficients, where $v$ is a preset small positive number. Otherwise, go to step 3. Step 3: Calculate the increment $\delta \boldsymbol{a}$ of the filter coefficient vector $\boldsymbol{a}_{k}=\left[a_{i k}(1), a_{i k}(2), \ldots, a_{i k}\left(N_{i}\right)\right]^{\mathrm{T}}$ at the $k$ th iteration in an $N_{i}$-dimensional linear space to minimise

$$
\left\|-\operatorname{Apx}\left(\boldsymbol{a}_{k}, \omega\right)-\delta \boldsymbol{a}^{\mathrm{T}} \nabla \operatorname{Apx}\left(\boldsymbol{a}_{k}, \omega\right)\right\|
$$

Utilising the PAS algorithm presented for solving the standard LP problem (36), we perform the following iterative procedure:

(3.1): Choose an initial guess $\boldsymbol{x}^{0}$ which satisfies the equality constraints $\boldsymbol{A}^{\mathrm{T}} \boldsymbol{x}^{0}=\boldsymbol{b}$ and $\boldsymbol{x}^{0}>0$ of (36). We can simply choose $\boldsymbol{x}^{0}=\left[\begin{array}{ll}x_{1}^{\mathrm{T}} & \boldsymbol{x}_{2}^{\mathrm{T}}\end{array}\right]^{\mathrm{T}}$, where $\boldsymbol{x}_{1}=\boldsymbol{x}_{2}=\mathbf{1} /(2 J), J$ is the number of sample points in frequency. Set the iteration number $m=0$.

(3.2): At the $m$ th iteration, compute $\boldsymbol{w}=\left[\begin{array}{ll}\delta \boldsymbol{a}^{\mathrm{T}} & \varepsilon\end{array}\right]^{\mathrm{T}}=$ $\left(\boldsymbol{A} \boldsymbol{D}_{x}^{2} \boldsymbol{A}^{\mathrm{T}}\right)^{-1} \boldsymbol{A} \boldsymbol{D}_{x}^{2} \boldsymbol{c}$ according to the following process:

(3.2.1): Construct two diagonal matrices $\boldsymbol{D}_{i}=\operatorname{diag}\left(\boldsymbol{x}_{i}\right)$, $i=1,2$.

(3.2.2): Compute $\boldsymbol{M}_{1}=\boldsymbol{D}_{1}^{2}+\boldsymbol{D}_{2}^{2}, \boldsymbol{M}_{2}=\boldsymbol{D}_{1}^{2}-\boldsymbol{D}_{2}^{2}, \mathrm{~g}=\mathbf{1}^{\mathrm{T}}$ $M_{1}^{2}$.

(3.2.3): Compute $\delta \boldsymbol{a}_{m}=\left(\boldsymbol{U}^{\mathrm{T}}\left(\boldsymbol{M}_{1}-\boldsymbol{M}_{2} \mathbf{1 1}^{\mathrm{T}} \boldsymbol{M}_{2} / \mathrm{g}\right) \boldsymbol{U}\right)^{-1} \times$ $\left(\boldsymbol{U}^{\mathrm{T}}\left(\boldsymbol{M}_{1}-\boldsymbol{M}_{2} \mathbf{1 1}^{\mathrm{T}} \boldsymbol{M}_{2} / \mathrm{g}\right) \boldsymbol{s}\right)$ and $\varepsilon=\left(\mathbf{1}^{\mathrm{T}} \boldsymbol{M}_{2}\left(\boldsymbol{U} \delta \boldsymbol{a}_{m}-\boldsymbol{s}\right)\right) / \mathrm{g}$.

Step 4: Compute $\varepsilon / \max \left\{\left|\boldsymbol{U} \delta \boldsymbol{a}_{m}-\boldsymbol{s}\right|\right\}$. Set the obtained $\delta \boldsymbol{a}_{m}$ equal to $\delta \boldsymbol{a}_{k}$ and go to step 7 if $\varepsilon / \max \left\{\left|\boldsymbol{U} \delta \boldsymbol{a}_{m}-\boldsymbol{s}\right|\right\} \geqq \eta$, where $\eta$ is a preset small positive number. Otherwise, go to step 5.

Step 5: Compute $\boldsymbol{d}_{x}=\boldsymbol{D}_{x}^{2} \boldsymbol{r}=\boldsymbol{D}_{x}^{2}\left(\boldsymbol{c}-\boldsymbol{A}^{\mathrm{T}} \boldsymbol{w}\right)$ according to the following process:

(5.1): Compute $\boldsymbol{d}_{1}=D_{1}^{2}\left(\boldsymbol{s}+\varepsilon \mathbf{1}-\boldsymbol{U} \delta \boldsymbol{a}_{m}\right), \boldsymbol{d}_{2}=\boldsymbol{D}_{2}^{2}(-\boldsymbol{s}+$ $\left.\varepsilon \mathbf{1}+\boldsymbol{U} \delta \boldsymbol{a}_{m}\right)$.

(5.2): Compute $\boldsymbol{d}_{x}=\left[\begin{array}{ll}\boldsymbol{d}_{1}^{\mathrm{T}} & \boldsymbol{d}_{2}^{\mathrm{T}}\end{array}\right]^{\mathrm{T}}$.

Step 6: Compute the step size $\xi / \gamma$ from (46) and update the primal variable vector according to

$$
\boldsymbol{x}^{m+1}=\boldsymbol{x}^{m}-\frac{\xi}{\gamma} \boldsymbol{d}_{x}
$$

Then, set $m=m+1$ and go to step 3.2.

Step 7: Use the optimal solution $\delta \boldsymbol{a}_{k}$ obtained to find the best increment such that

$$
\left\|\operatorname{Apx}\left(\boldsymbol{a}_{k}+\beta \delta \boldsymbol{a}_{k}, \omega\right)\right\|, \forall \beta \geqq 0
$$

is minimised. We adopt the Nelder and Mead simplex algorithm [20] to perform a line search to find the best value of $\beta$. Let the best value of $\beta$ be $\beta_{k}$. We update the filter coefficient vector according to $\boldsymbol{a}_{k+1}=\boldsymbol{a}_{k}+\beta_{k} \delta \boldsymbol{a}_{k}$. Then, set $k=k+1$ and go to step 2 .

\section{Simulation results}

In this Section, we present simulation results for the design of two-channel linear-phase QMF banks based on real IIR DAFs for illustration and comparison. These designs were performed on a personal computer with Pentium-III CPU using the MATLAB programming language. For comparison, design results using the proposed technique and using techniques presented in [11] and [12] are presented. The performance for each of the QMF banks designed is evaluated in terms of peak stopband ripple (PSR) of 
$H_{0}(z)$, maximal variation of phase response (MVPR) and maximal variation of group delay (MVGD) in $T\left(\mathrm{e}^{\mathrm{j} \omega}\right)$, and maximal variation of filter-bank response (MVFBR). They are defined as follows:

$$
\begin{aligned}
& \operatorname{PSR}=20 \log _{10}\left(\max _{\omega_{l} \in\left[\omega_{s}, \pi\right]}\left|H_{0}\left(\mathrm{e}^{\mathrm{j} \omega_{l}}\right)\right|\right)(\mathrm{dB}) \\
& \operatorname{MVPR}=\max _{\omega_{l} \in[0, \pi]} \mid \operatorname{Arg}\left\{T\left(\mathrm{e}^{\mathrm{j} \omega_{l}}\right)\right\} \\
& +\left(2 N_{1}+2 N_{2}+1\right) \omega_{l} \mid(\text { radians }) \\
& \text { MVGD } \max _{\omega_{l} \in[0, \pi]} \mid G D\left\{T\left(\mathrm{e}^{\mathrm{j} \omega_{l}}\right)\right\} \\
& -\left(2 N_{1}+2 N_{2}+1\right) \mid(\text { samples }), \\
& \operatorname{MVFBR}=\max _{\omega_{l} \in[0, \pi]}\left(20 \log _{10}\left|T\left(\mathrm{e}^{\mathrm{j} \omega_{l}}\right)-\frac{1}{2} \mathrm{e}^{-\mathrm{j}\left(2 N_{1}+2 N_{2}+1\right) \omega_{l}}\right|\right)(\mathrm{dB})
\end{aligned}
$$

where $G D(x)$ denotes the group delay of $x$. The performance for each of the QMF banks designed using the techniques of [11] and [12] are evaluated based on the filter coefficients presented in [11] and [12].

Example 1: This example is the same as that given in ([11], Ex. 1). We use the same specifications for this design: real IIR DAFs $A_{1}\left(z^{2}\right)$ and $A_{2}\left(z^{2}\right)$ with orders $N_{1}$ and $N_{2}$ equal to 3 and 2 , respectively, low-pass analysis filter $H_{0}(z)$ with passband edge frequency $\omega_{p}=0.4 \pi$ and stopband edge frequency $\omega_{s}=0.6 \pi$. Significant design results, namely, PSR, MVPR, MVGD, and MVFBR obtained using the technique of [11] and the proposed technique are shown in Table 1 for comparison. Table 2 lists the filter coefficients obtained after three iterations for both designs of $A_{1}\left(z^{2}\right)$ and $A_{2}\left(z^{2}\right)$, using the proposed technique with parameters $\xi=0.97, \eta=0.99999$, and $L=8\left(N_{i}+1\right)$. The parameter $v$ was set to $2 \times 10^{-8}$ to design $A_{1}\left(z^{2}\right)$ and $4 \times 10^{-6}$ to design $A_{2}\left(z^{2}\right)$. The corresponding magnitude responses of the $H_{0}\left(\mathrm{e}^{\mathrm{j} \omega}\right)$ designed are shown in Fig. 4. The resulting phase errors and group delay deviations of the QMF banks designed are depicted in Figs. 5 and 6, respectively. Figure 7 plots the variations of the designed filter-bank responses. Simulation results show that the proposed technique

Table 1: Significant design results for design example 1

\begin{tabular}{lcr}
\hline & Proposed technique & Technique of [11] \\
\hline PSR (dB) & -19.965154415 & -18.051817634 \\
MVPR (radians) & 0.205953001 & 0.303082474 \\
MVGD (samples) & 1.497994713 & 2.161264942 \\
MVFBR (dB) & -19.760593942 & -16.422653793 \\
\hline
\end{tabular}

Table 2: Filter coefficients designed for design example 1

\begin{tabular}{lrr}
\hline$n$ & \multicolumn{1}{c}{$a_{1}(n)$} & \multicolumn{1}{c}{$a_{2}(n)$} \\
\hline 0 & 1.00000000000000 & 1.00000000000000 \\
1 & 0.23809492090228 & -0.23721170338893 \\
2 & -0.07300653565757 & 0.15616284193843 \\
3 & 0.03862697338297 &
\end{tabular}

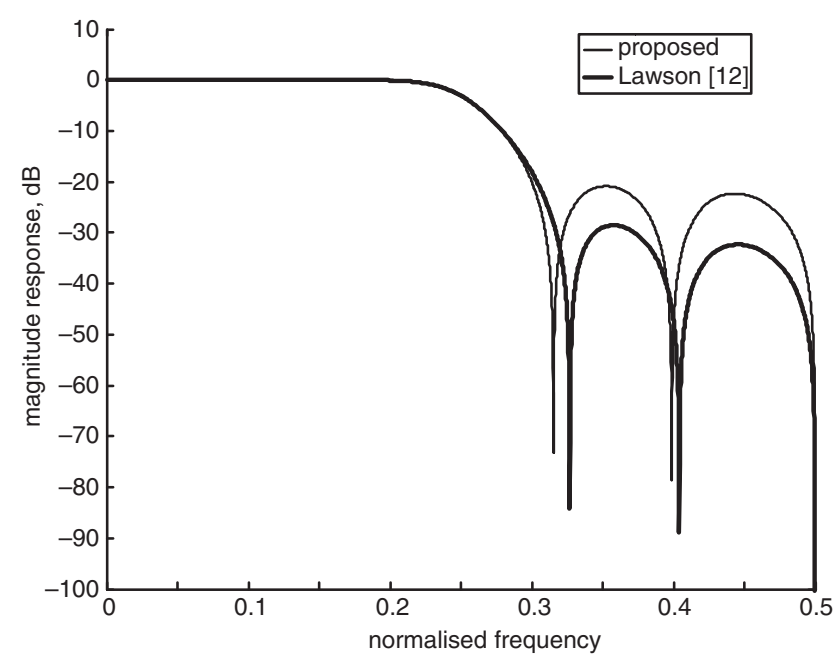

Fig. 4 Magnitude responses of low-pass analysis filters designed for example 1

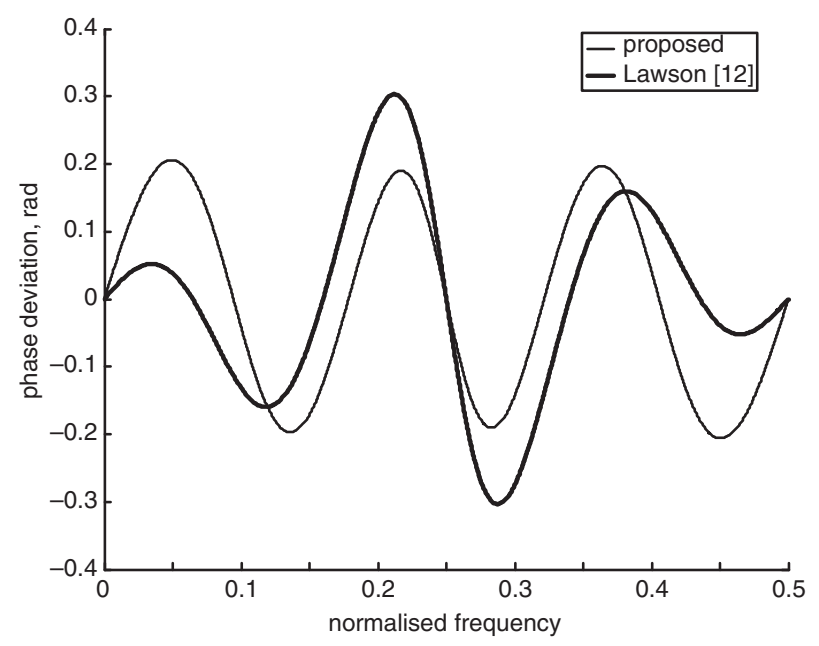

Fig. 5 Phase deviations of QMF banks designed for example 1

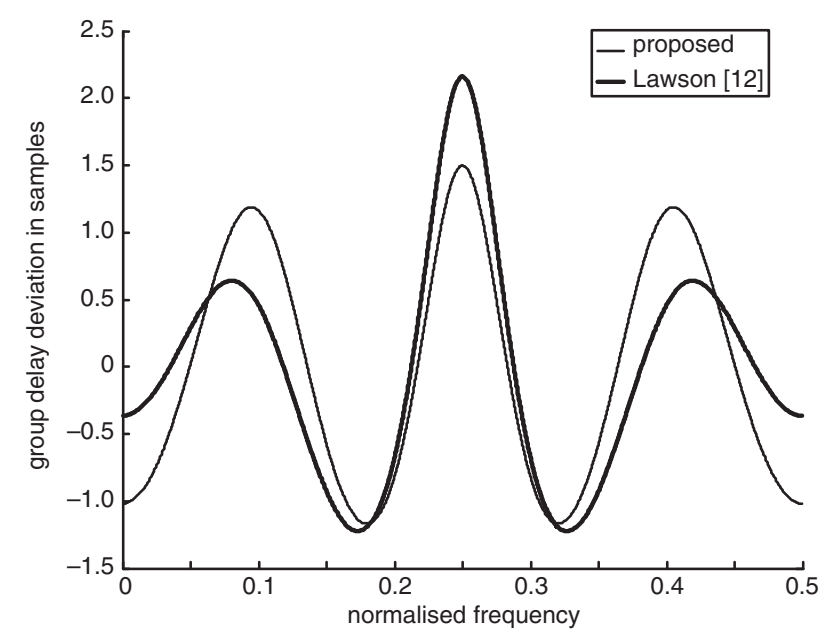

Fig. 6 Group delay deviations of QMF banks designed for example 1

provides very satisfactory design results when compared to the technique of [11].

Example 2: This example is the same as ([12], Ex. 1). We use the same specifications for this design: real IIR DAFs $A_{1}\left(z^{2}\right)$ and $A_{2}\left(z^{2}\right)$ with orders $N_{1}=N_{2}=2$, low-pass analysis filter $H_{0}(z)$ with passband edge frequency 


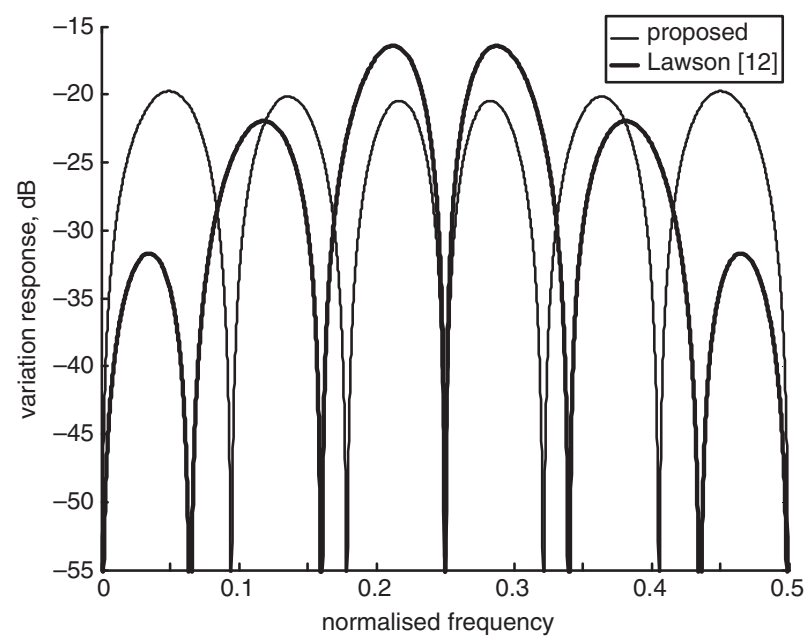

Fig. 7 Variations of filter bank responses designed for example 1

Table 3: Significant design results for design example 2

\begin{tabular}{lrr}
\hline & Proposed technique & Technique of [12] \\
\hline PSR (dB) & -18.112979613 & -15.617494878 \\
MVPR (radians) & 0.132845763 & 0.198064914 \\
MVGD (samples) & 0.895850891 & 1.212561067 \\
MVFBR (dB) & -23.560033741 & -20.098651279
\end{tabular}

Table 4: Filter coefficients designed for design example 2

\begin{tabular}{lrr}
\hline$n$ & \multicolumn{1}{c}{$a_{1}(n)$} & \multicolumn{1}{c}{$a_{2}(n)$} \\
\hline 0 & 1.00000000000000 & 1.00000000000000 \\
1 & -0.23721170339174 & 0.23401447596825 \\
2 & 0.15616284195027 & -0.09173259794723
\end{tabular}

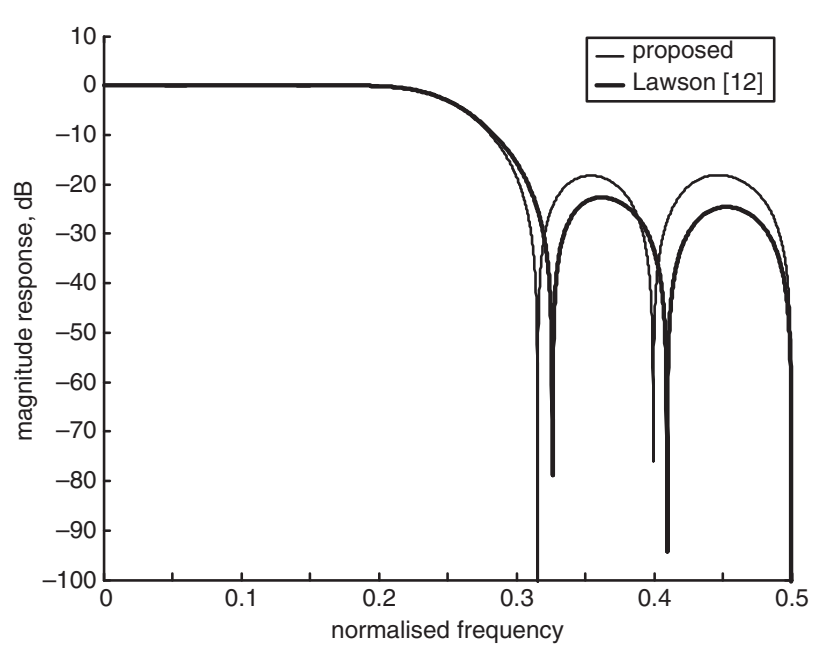

Fig. 8 Magnitude responses of low-pass analysis filters designed for example 2

$\omega_{p}=0.4 \pi$ and stopband edge frequency $\omega_{s}=0.6 \pi$. Significant design results, namely, PSR, MVPR, MVGD, and MVFBR obtained by using the technique of [12] and the proposed technique are shown in Table 3 for comparison. Table 4 lists the filter coefficients obtained after four iterations and three iterations for the designs of $A_{1}\left(z^{2}\right)$ and $A_{2}\left(z^{2}\right)$, respectively, using the proposed technique with parameters $\xi=0.97, \eta=0.99999$, and $L=8\left(N_{i}+1\right)$.

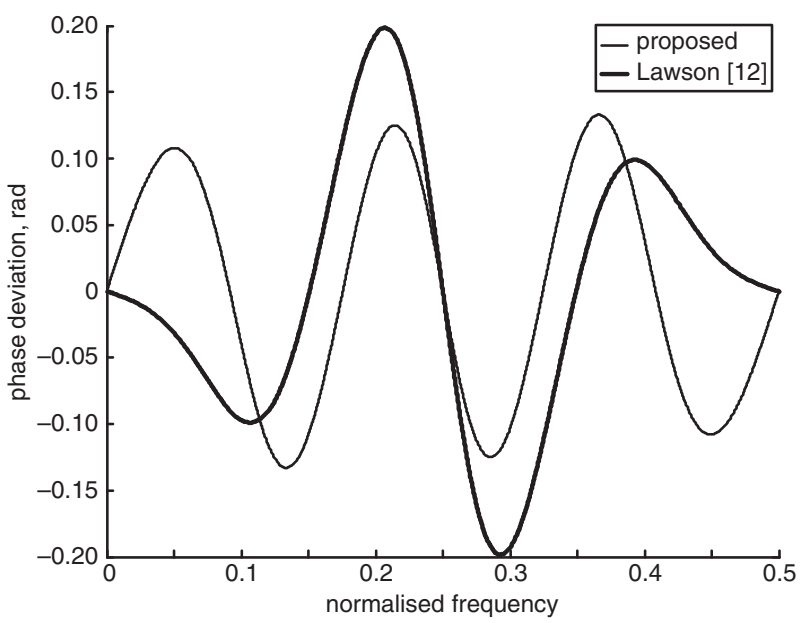

Fig. 9 Phase deviations of QMF banks designed for example 2

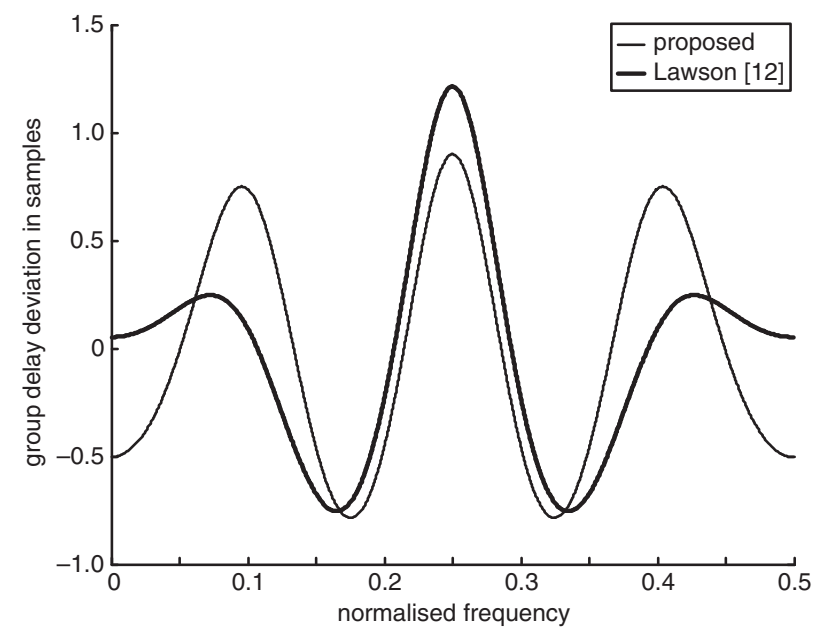

Fig. 10 Group delay deviations of QMF banks designed for example 2

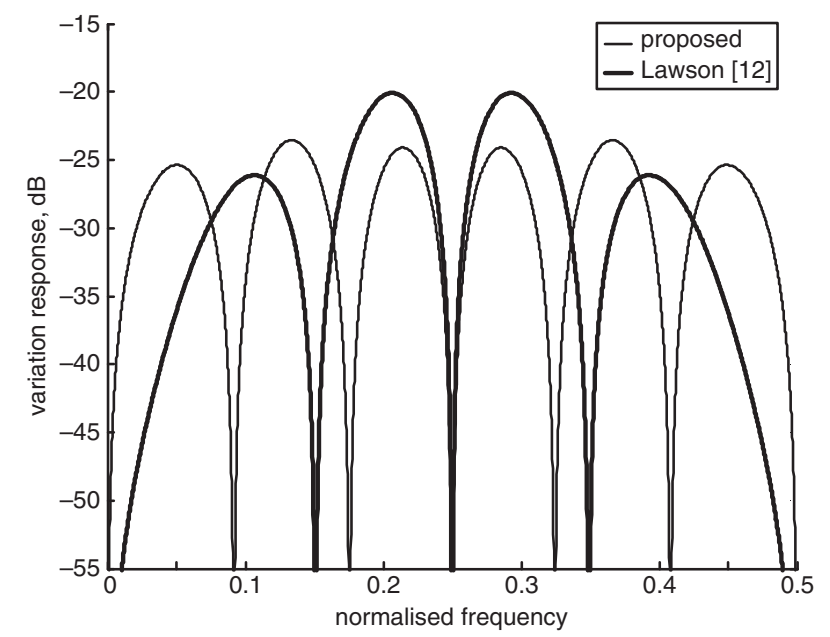

Fig. 11 Variations of filter bank responses designed for example 2

The parameter $v$ was set to $2 \times 10^{-8}$ to design $A_{1}\left(z^{2}\right)$ and $4 \times 10^{-6}$ to design $A_{2}\left(z^{2}\right)$. The corresponding magnitude responses of the $H_{0}\left(\mathrm{e}^{\mathrm{j}} \omega\right)$ designed are shown in Fig. 8. The resulting phase errors and group delay deviations of the QMF banks designed are depicted in Figs. 9 and 10, respectively. Figure 11 plots the variations of the designed filter-bank responses. Simulation results show that the proposed technique provides much better performance than the technique of [12]. 


\section{Conclusions}

This paper has presented a technique for the design of twochannel linear-phase quadrature mirror filter (QMF) banks based on real infinite impulse response (IIR) digital all-pass filters. The design problem is first formulated as a nonlinear optimisation problem of an appropriate objective function for the phase response using the minimax $\left(L_{\infty}\right)$ error criteria. A nonlinear minimax algorithm is used to generate a sequence of linear Chebyshev approximation problems. Solving each of the linear Chebyshev approximations provides the required increment for updating the filter coefficients during each iteration. This leads to the linear Chebyshev approximation of the desired response from a linear subspace related to the objective function, which can easily be solved using a variant of Karmarkar's algorithm. Computer simulations have shown the effectiveness of the proposed technique.

\section{Acknowledgments}

This work was supported by the National Science Council under Grant NSC90-2213-E002-096.

\section{References}

1 Crochiere, R.E.: 'Digital signal processor: sub-band coding', Bell Syst. Tech. J., 1981, 60, pp. 1633-1653

2 Bellanger, M.G., and Daguet, J.L.: 'TDM-FDM transmultiplexer: digital polyphase and FFT', IEEE Trans. Commun., 1974, 22, pp. 1199-1204

3 Vary, P., and Heute, U.: 'A short-time spectrum analyzer with polyphase network and DFT', Signal Process., 1980, 2, pp. 55-65

4 Woods, J.W., and O'Neil, S.D.: 'Subband coding of images', IEEE Trans. Acoust. Speech Signal Process., 1986, 34, pp. 1278-1288

5 Basu, S., Chiang, C., and Choi, H.: 'Wavelets and perfect reconstruction coding with causal stable IIR filters', IEEE Trans. Circuits Syst. II, Analog Digit. Signal Process., 1995, 42, pp. 24-38
6 Ekanayake, M.M., and Premaratne, K.: 'Two-channel IIR QMF banks with approximately linear-phase analysis and synthesis filters', IEEE Trans. Signal Process., 1995, 43, pp. 2313-2322

7 Creusere, C.D., and Mitra, S.K.: 'Image coding using wavelets based on perfect reconstruction IIR filter banks', IEEE Trans. Circuits Syst. Video Technol., 1996, 6, pp. 447-458

8 Argenti, F., Cappellini, V., Sciorpes, A., and Venetsanopoulos, A.N.: 'Design of IIR linear-phase QMF banks based on complex allpass sections', IEEE Trans. Signal Process., 1996, 44 pp. $1262-1267$

9 Lu, C.-K., Anderson, M., and Summerfield, S.: 'Design of approximately linear-phase allpass based QMF banks'. Proc. Int. Symp. on Digital signal processing, 1996, London, pp. 56-61

10 Summerfield, S., and Lu, C.-K.: 'Design and VLSI implementation of multirate filter banks based on approximately linear phase allpass sections'. Proc. Int. Symp. on Circuits and systems, Monterey, CA, 1998, pp. $413-416$

11 Lawson, S.S., and Klouche-Djedid, A.: 'Technique for design of twochannel approximately linear phase QMF bank and its application to image compression', IEE Proc., Vis. Image Signal Process., 2001, 148 (2), pp. $85-92$

12 Lawson, S.S.: 'Design of IIR-based wavelet filter banks and their application to image coding'. Proc. IEE Seminar on Time-scale and time-frequency analysis and applications, 2000 pp. $7 / 1-7 / 6$

13 Lee, J.-H., and Niu, I.-C.: 'Minimax design of two-channel IIR QMF banks with arbitrary group delay', IEE Proc., Vis. Image Signal Process., 2001, 148, (6), pp. 384-390

14 Vaidyanathan, P.P., Regalia, P.A., and Mitra, S.K.: 'Design of doublycomplementary IIR digital filters using a single complex allpass filter, with multirate applications', IEEE Trans. Circuits Syst., Apr. 1987, 34, pp. $378-388$

15 Ikehara, M., Funaishi, M., and Kuroda, H.: 'Design of complex all-pass networks using Remez algorithm', IEEE Trans. Circuits Syst. II, Analog Digit. Signal Process, Aug. 1992, 39, pp. 549-556

16 Adler, I., Karmarkar, N., Resende, M.G.C., and Veiga, G.: 'An implementation of Karmarkar's algorithm for linear programming', Math. Program., 1989, 44, pp. 297-335

17 Osborne, M.R, and Watson, G.A.: 'An algorithm for minimax approximation in the nonlinear case', Comput. J., 1969, 12, pp. 63-68

18 Vaidyanathan, P.P.: 'Multirate systems and filter banks' (Prentice-Hall, Englewood Cliffs, NJ, USA, 1992)

19 Papadimitriou, C.H., and Steiglitz, K.: 'Combinatorial optimization: algorithms and complexity' (Prentice-Hall, Englewood Cliffs, NJ, USA, 1982)

20 Nelder, J.A., and Meade, R.: 'A simplex method for function minimization', Comput. J., 1965,7 , pp. 308-313 